\title{
Performance Estimation of Static Grid Octagonal Topology under Distributed Environment through Simulation Tool
}

\author{
Vipin Saxena, Ph. D \\ Department of Computer Science \\ B.B. Ambedkar University \\ (A Central University) \\ Rae Barely Road, Lucknow-25, U.P. (India)
}

\author{
Nimesh Mishra \\ Department of Computer Science \\ B.B. Ambedkar University \\ (A Central University) \\ Rae Barely Road, Lucknow-25, U.P. (India)
}

\begin{abstract}
In the recent year, distributed computing systems are one of most efficient and a very popular approach of computing as compared to the old centralized computing systems. Such network processing environment provides a very high performance computing in a very low cost. Once such computing implemented in proposed topological architecture for static interconnection, achieves the desired accuracy. The present work is an attempt to measure the performance of different types of traffic such as video conferencing, file transfer as well as email service through the newly proposed octagonal topology. An application of the OPNET simulation tool is executed using packets transmission through octagonal grid network topology. Analysis of different types of applications packets has been done in the present work and study has been represented using graphs.
\end{abstract}

\section{General Terms}

Octagonal Grid Topology, Performance, Packets

\section{Keywords}

Distributed Computing, Octagonal Grid Topology, Packet Transmission, Simulation, Opnet

\section{INTRODUCTION}

The network topology plays a very vital role in the static interconnection of computer systems, hand-held devices like laptop's mobile devices, etc. In the present work, the search of optimum path for transferring of data packets from source to destination end has been done to compute shortest path. Designing of network topology for data transmission, is a critical issue, related to the reliability, fast data transmission, authentication, fault tolerance and it should have support of running multiple services. The proposed topology consists of multipath route for data transmission in a reliable manner. The motive behind designing such a system is mainly to fulfill the requirements of the network users. But such design may cause a huge loss of cost if it is not according to the need of the users. Thus, if it is pre-tested using any simulation tool to check its performance issues then it can reduce risk of cost wastage. Thus, in order to check the efficiency of the proposed topology, a simulation tool OPNETTM IT Guru Academic edition software, has been used. This will restrict and save the cost as it will results in removing if and buts of the proposed topology. The present paper is based upon the computation of performance of octagonal topology through OPNET (Optimized Packet Network Engineering Tool) and further data packets dropped rate, data packet received, ethernet delay, packets collisions, etc, are computed from the same tool for the proposed topology.

\section{RELATED WORK}

Hwang [1] explained static and dynamic type of the topologies along with the complexity. Static interconnection like bus, ring, mesh, fully connected tree, etc are explained and further dynamic interconnection like hypercube, digital bus, multistage network, crossbar network etc are defined, along with the complexity comparison. Tanenbaum [2] has deeply discussed the design of network system including the different types of protocol explanation. The peer-to-peer and broadcast communications are explained. Network traffic loading and balancing issues are also explained. Liu [3] has discussed the distributed computing approach of process execution in the computer network system. Different types of distributed computing environment paradigm have been designed for assistance of the execution of the distributed processing in the network. In the current scenario distributed approach is more important over the centralized approach because it connects a collection of heterogeneous devices within a low budget. Bates [4] had proposed a debugging tool for the evaluation of bugs in the heterogeneous computer network systems. From this tool one can easily predict the bugs occurred in the data transmission throughout the node communication. Garousi et al. [5] have studied the real time systems to check the occurrence of stress in the packets transformation. This traffic stress is the testing implementation in the distributed computing environment unbalancing aware's the users to check the load on the network. Huang and Bode [6] have performed a comparative study of the performance in the ring and the tree topology. They had worked to give preference to the best performance topology design for the network topology based on the performance issue. Kamalesh and Srivastava [7-8] have worked out the design techniques for finding minimum cost from source to destination are well explained for the different types of topology used for the survival network. The designing of the network architecture, is one of the important issues in the heterogeneous network for the distributed computing systems and these factors affect the throughput or rate of utilization of the processors. Different types of topologies are modeled by the use of object oriented unified modeling language for the distributed computing system by Saxena and Arora [9]. Singh et al. [10] have proposed a heuristic approach for load sharing for the distributed computing system. The processor utilization is directly proportional to the performance estimation. Real time distributed system is a complex system in the current scenario, for example, aircraft simulation is based on the distributed 
approach of computing system. These aspects are well explained in distributed computing environment.

\section{BACKGROUND}

\subsection{Traffic Generation}

In a computer network systems environment, communication takes place between the sender nodes to the receiver nodes in the form of signals. The data in the form of these signals becomes a true traffic for the users. This paper presents a complete network analyzer development for heterogeneous services in proposed octagonal network topology. A network analyzer is also called a packet analyzer, "traffic analyzer" and "protocol analyzer". Different types of services like Video Conferencing, Voice over IP and FTP application are running on the network generate traffic which generally has to face several issues, which becomes a measure of performance of network. These performance issues are throughput, packets received, packets transformed, collisions, delay, jitter, throughput, and error rates, etc. It is very essential to get a somehow prediction of performance issues of network traffic using any method which may be manually if possible or using any of available tool before the actual design of such network to reduce the wastage of cost factor of topological design. An OPNET tool is used to test on traffic and utilization generated by the several services. One can test the reliability of network analyzer by its implementation over Video conferencing, VoIP and FTP services. There is also need of management of the topological structure in terms of monitoring, configuration, troubleshooting of the network elements like hosts, routers, switches, server and connecting links. In order to ensure that the selected simulation tool meets the network performance requirements and compatibility issues, the problem is experimentally tested using the 'OPNET' tool for performance of VoIP, Video-Conferencing and ftp services.

\subsection{OPNET Simulation Tool}

OPNET allows a user to simulate any network formed by different types of network devices like routers, switches, nodes and different types of communication links between the devices having different configuration as needed by the network administrator and users. The OPNET Technologies, Inc. has offered a network traffic simulation tool for the enterprises and the universities and is very useful for creating a simulated network and learning the fundamentals of networking technologies. This tool provides easy evaluation of several network performance issues also like channel utilization, throughput, offered load, channel capacity, message size, interarrival, message/frame/ethernet/etc delay, dropped frame, collision, etc. The functioning is based on the concept of designing of network architecture using well configured devices and then to create scenarios containing different types of traffic flows and then calculates the discussed parameters of traffic control.

\section{EXPERIMENTAL STUDY}

The proposed octagonal network topology configuration has been designed, using OPNET Tool. The network setting consists of $\mathrm{MxN}$ architecture of octagonal topology using 10 baseT link, and 20 number of router's. Along with this a FTP server (that supports FTP and file print services) is connected to one of the edge router via the 10 baseT link. All the router nodes of the grid are connected in octagonal ring structure form using a 100 baseT link. Routers themselves have been classified into two groups one is edge router group present at surfaces area of the proposed topology and others are core routers placed at interior areas of the proposed topology. Edge routers provide their services to the end user's while core routers communicate among themselves to establish a path for transferring different types of packets from source edge router to destination edge router. The proposed topology is shown in figure 1 contains $\mathrm{ftp}$ server, video server, and voice server with total number of 20 nodes.

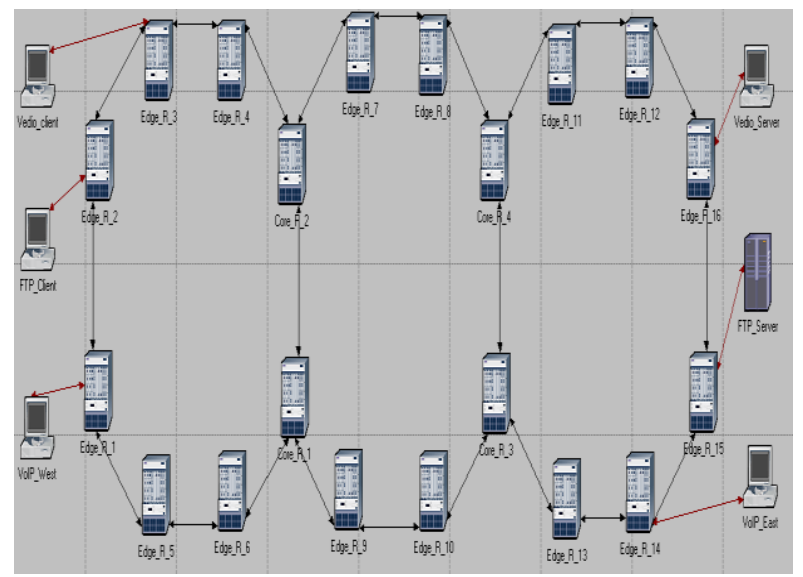

Fig 1: Octagonal Topology using OPNET

Video conferencing application using Video_Client and a Video_Server node connected using 10 baseT link to the edge routers and a VoIP application by connecting VoIP nodes at two different regions in this network takes place. The performance is measured and can be extended for the MxN network. In a file transfer protocol between the client and server, the maximum segment is taken as $3.5 \mathrm{~m}$ and the client load is 49,000 approx. At the site of server the rate of packet received is up to maximum 3.0. The Ethernet load at the server site is up to maximum of 5.5 packets / $\mathrm{sec}$. Delay is also computed at the end of client which is varying from test data 0.0090 to 0.0108 which is measured in the seconds. These are depicted in figures 2, 3, 4 and 5 .

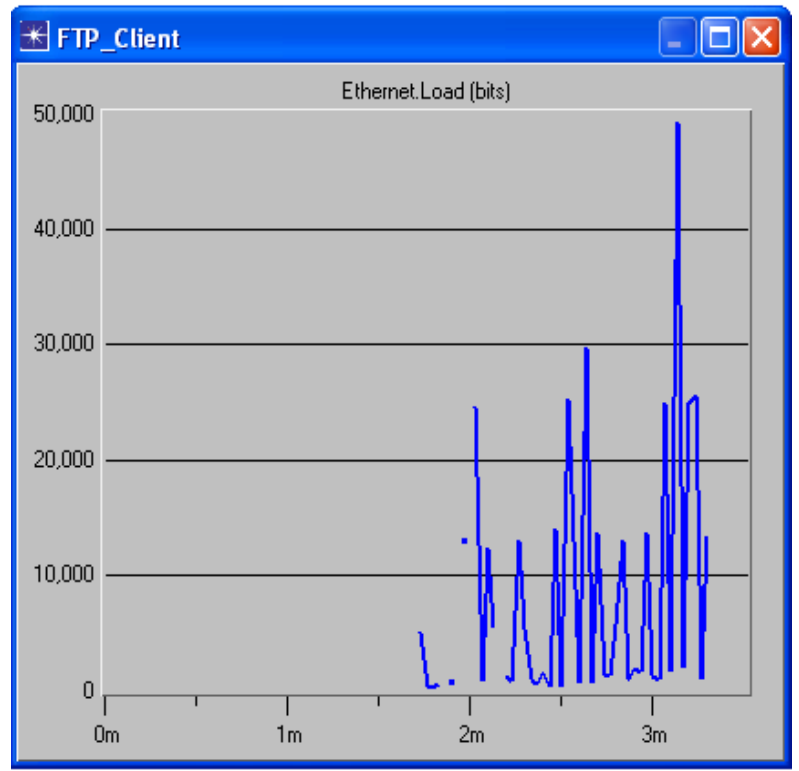

Fig 2: Ethernet Load (bits) in FTP Application 


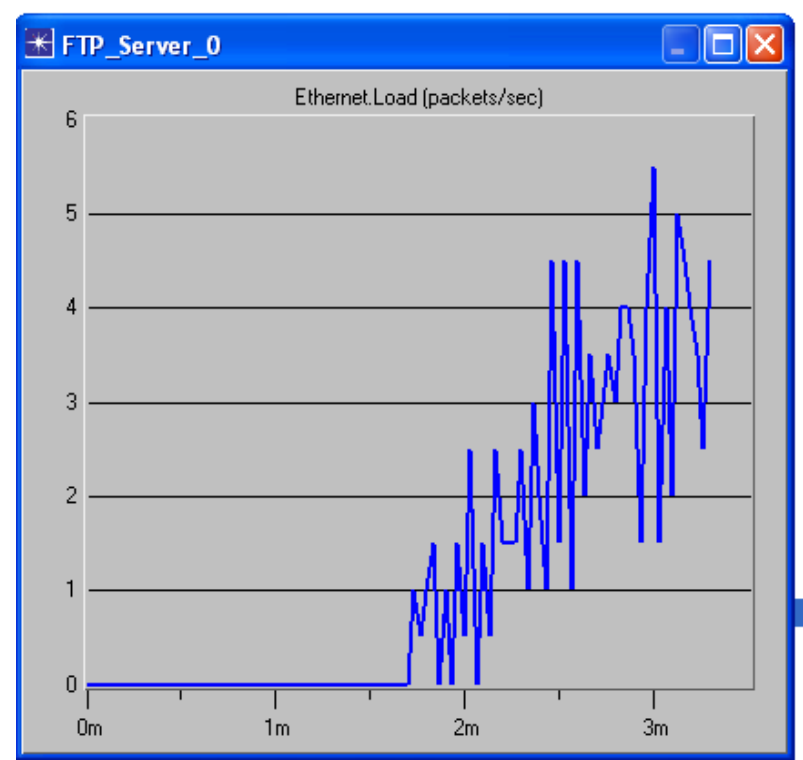

Fig 3: Ethernet Load (packets/sec) in FTP Application

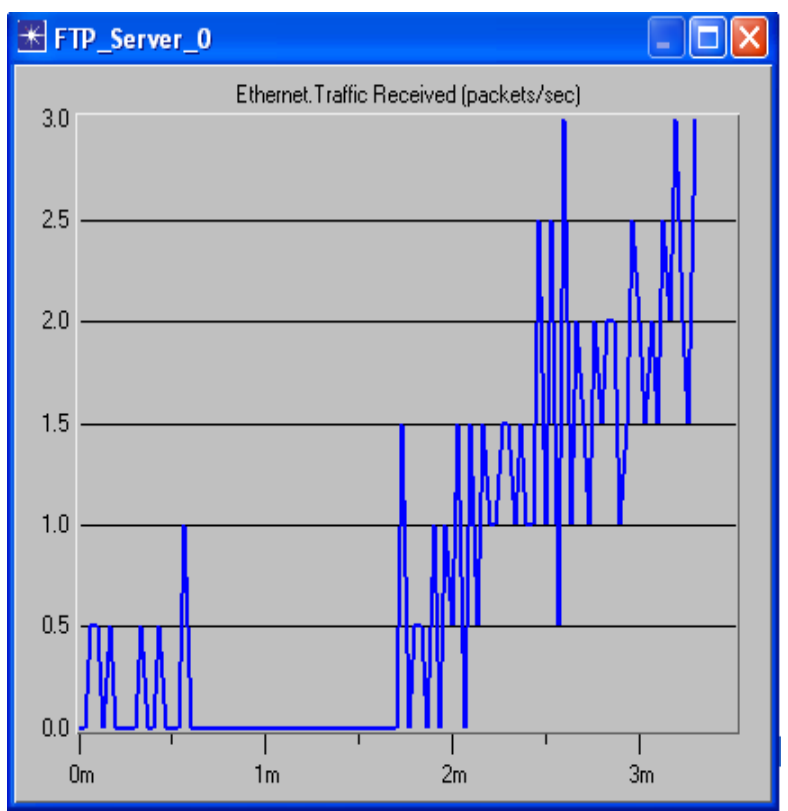

Fig 4: Ethernet Traffic Received (packets/sec) in FTP Application

Now let us increase the size of packets by considering the video files. The load is represented in figure 6 which goes up to maximum of 260 packets per seconds.

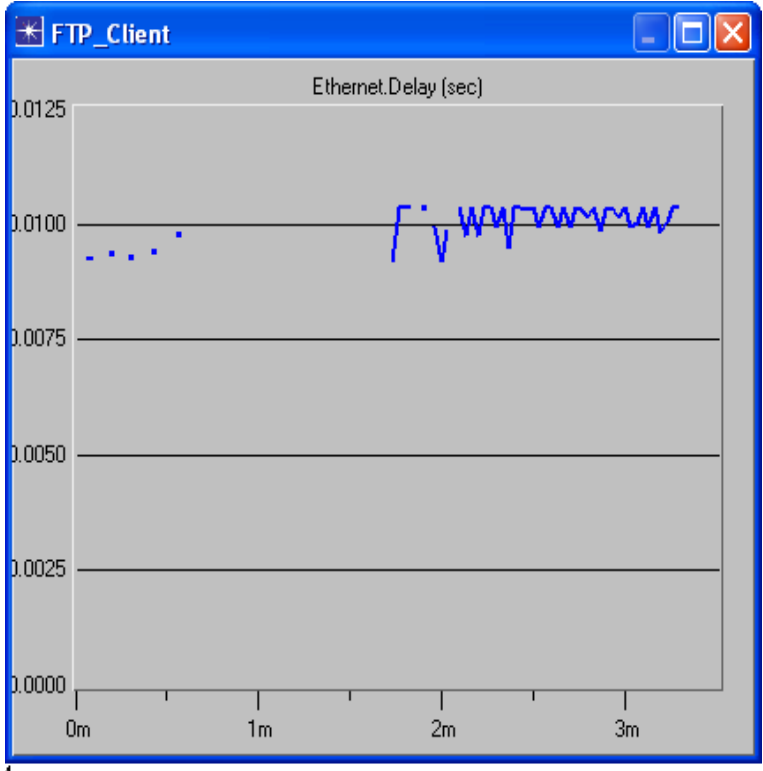

Fig 5: Ethernet Delay (sec) in FTP Application

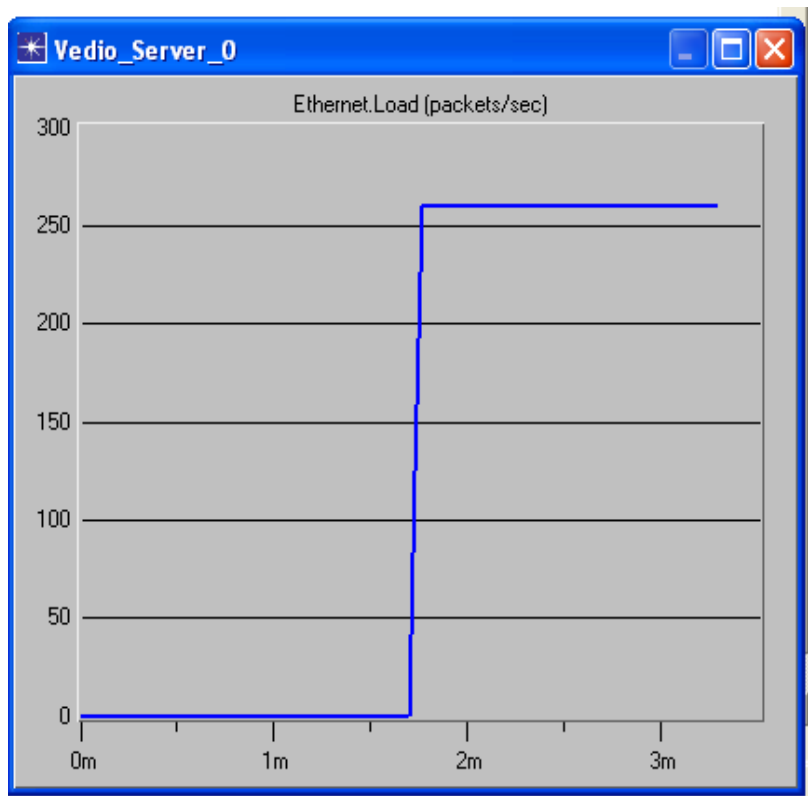

Fig 6: Ethernet Load (packets/sec) in Video Application

The file is transferred for conferencing purpose on the video server for which maximum data received is 137.5 packets/sec as represented in figure 7. 


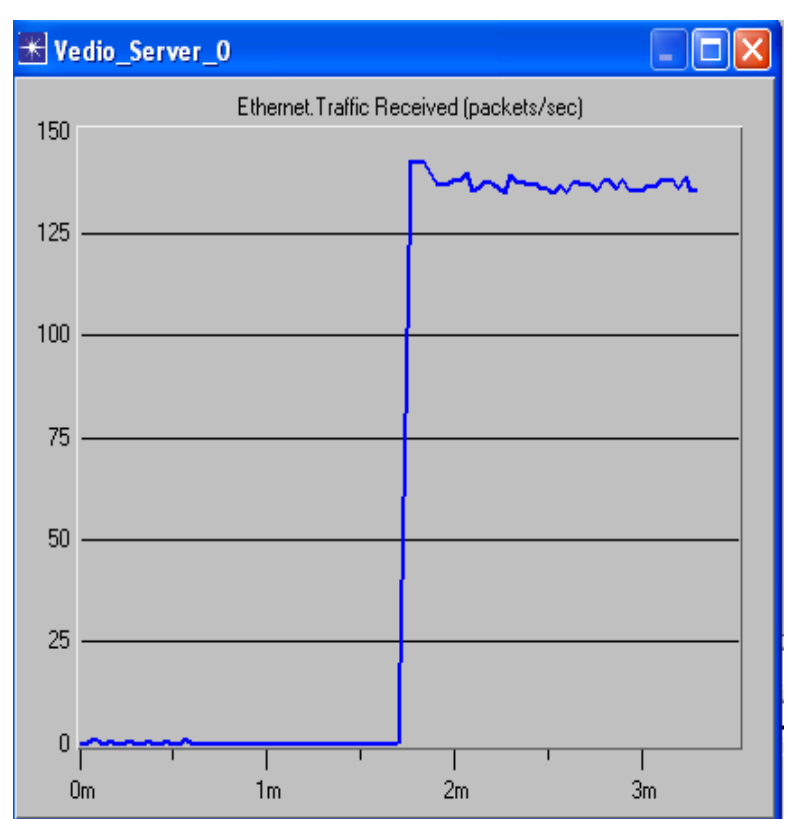

Fig 7: Ethernet Traffic Received (packets/sec) in Video Application

The traffic resend for the video conferencing is maximum upto 390,000 bytes per second as shown in figure 8 .

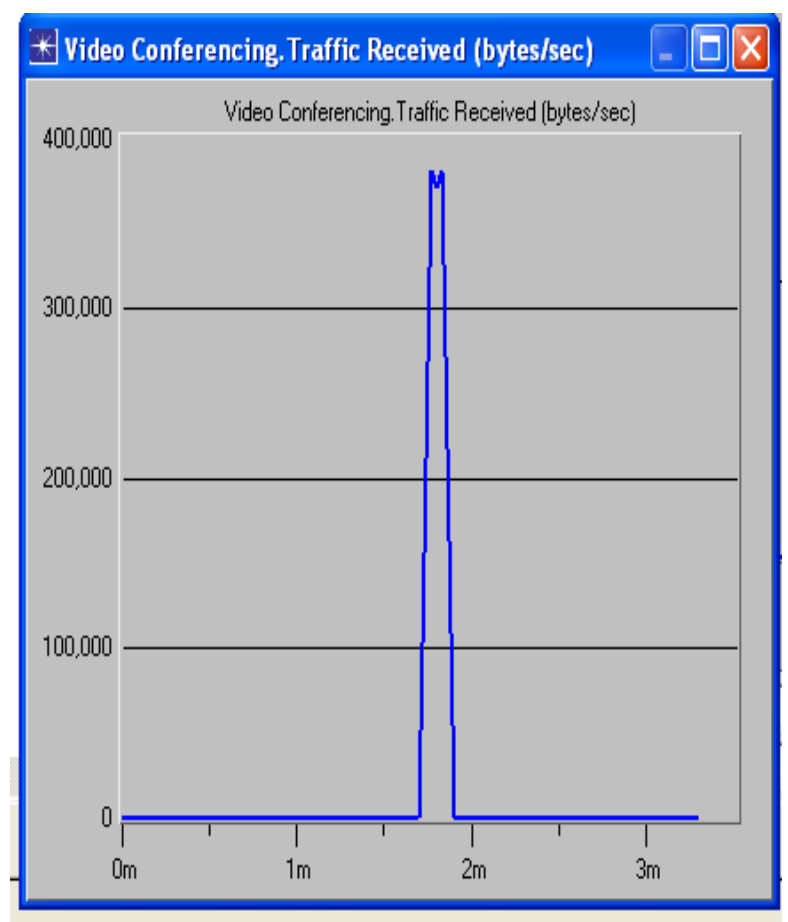

Fig 8: Video Conferencing Traffic Received (bytes/sec)

The delay is also computed at the video server site which goes up to maximum of 0.0095 secs as represented in the figure 9 .

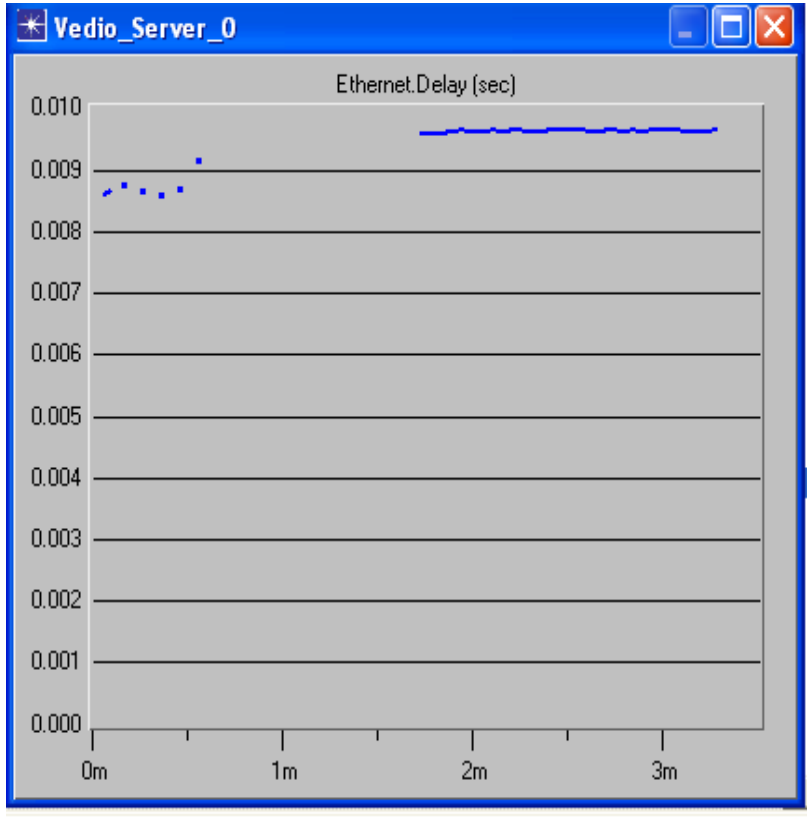

Fig 9: Ethernet Delay (sec) in Video Application

Voice files are also transmitted for the computation of delay variation. The maximum bytes for voice traffic received is 32,000 bytes per/ sec for a cable segment of 3.5 as shown in figure 10 .

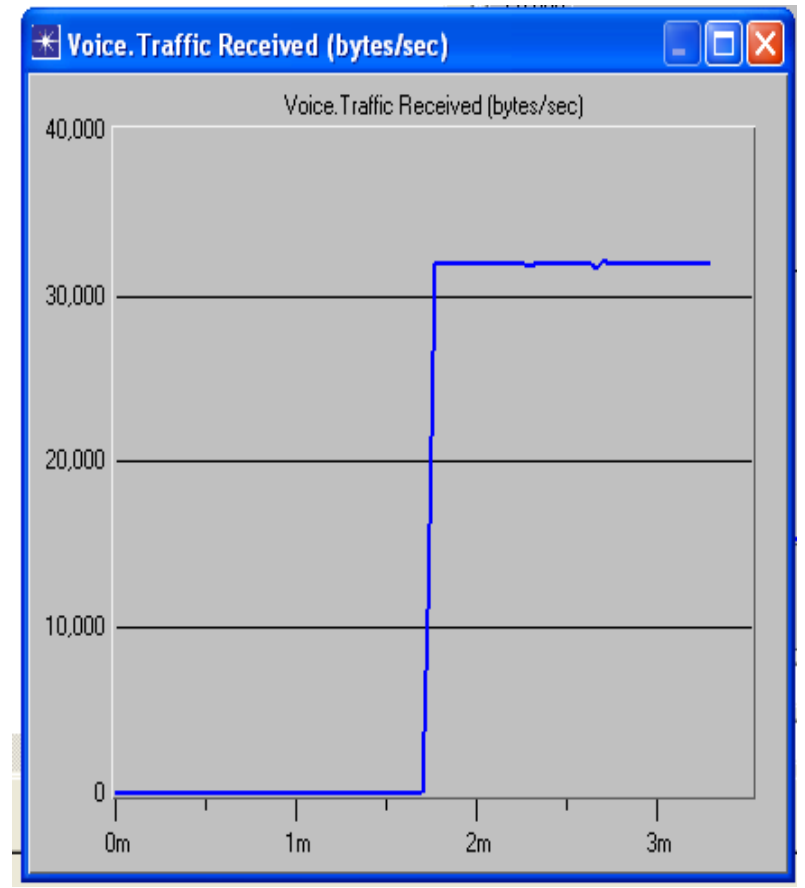

Fig 10: Voice Traffic Received (bytes/sec)

The voice packet delay variation is represented in figure 11 which goes up to 0.5 secs. 


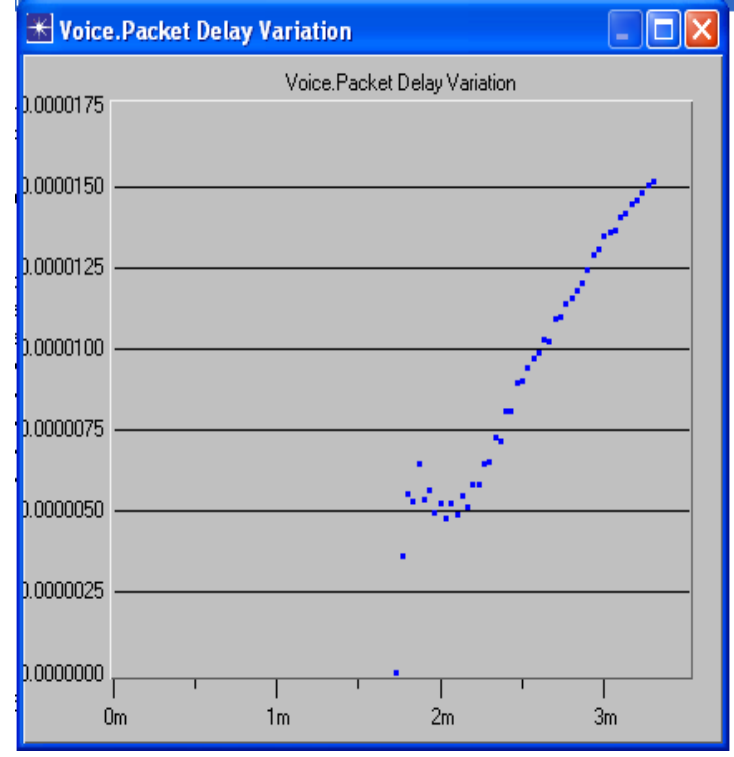

Fig 11: Voice Packet Delay Variations

\section{CONCLUSIONS}

The concept of graph theory or chemical structure can be used for designing of new network topology which should be reliable, robust, cost minimization etc, and these are the key features in the above work. Limited 20 nodes are considered for the new static octagonal topology which may be extended in future according to designed numbers and as per increase of octets. Loading and balancing are the important issues when one transfers the files from source end to the destination end. An important tool OPNET is used for getting the results related to network traffic loading packet received, delay related to voice and video files which have large size in comparison of text files. The delay in transmission is very less which shows that the proposed topology is reliable and robust.

\section{REFERENCES}

[1] Hwang, K., 2004, Advance Computer Architecture, 4th ed, Tata McGraw Hill.
[2] Tanenbaum, A.S., 2002, Computer Networks, 4th ed., Prentice Hall Publications.

[3] Liu, M.L., 2009, Distributed Computing Principles and Applications, 4th ed., Pearson Education.

[4] Bates, P., 1988, Distributed Debugging Tools for Heterogeneous Distributed Systems, International Conference, pp. 308-315.

[5] Garousi, V., Briand, L.C. and Labiche, Y., 2008, Traffic Aware Stress Testing of Distributed Real Time Systems Based on UML Models Using Genetic Algorithms, Journal of Systems and Software, Vol. 81, No. 2, pp.161185 .

[6] Huang, M. and Bode, B., 2005, A Performance Comparison of Tree and Ring Topologies in Distributed Systems, In Proceedings of the 19th IEEE International Parallel and Distributed Processing Symposium (Ipdps'05)-Workshop 13, Vol. 14, IPDPS, Computer Society, Washington, DC, pp. 258.1.

[7] Kamalesh, V.N. and Srivatsa, S.K., On the Assignment of Node Number in a Computer Communication Network, Proceedings of the World Congress on Engineering and Computer Science 2008, WCECS 2008, October 22-24, 2008, San Francisco, USA.

[8] Kamalesh, V.N. and Srivatsa, S.K., On the Design of Minimum Cost Survivable Network Topologies, NCC 2009, January 16-18,IIT Guwahati.

[9] Saxena,,V. and Arora. D., UML Modeling of Network Topologies for Distributed Computer System, Journal of Computing and Information Technology - CIT 17, 2009, 4, pp. 327-334.

[10] Singh, Y.J., Singh, Y.S., Gaikwad, A. and Mehrotra, S.C., Dynamic management of transactions in distributed real-time processing system, International journal of database management systems (IJDMS), vol.2, No.2, May 2010. 